\title{
Dynamics of alpha oscillations elucidate facial affect recognition in schizophrenia
}

\author{
Tzvetan G. Popov • Brigitte S. Rockstroh • Petia Popova • \\ Almut M. Carolus • Gregory A. Miller
}

\begin{abstract}
Impaired facial affect recognition is characteristic of schizophrenia and has been related to impaired social function, but the relevant neural mechanisms have not been fully identified. The present study sought to identify the role of oscillatory alpha activity in that deficit during the process of facial emotion recognition. Neuromagnetic brain activity was monitored while 44 schizophrenia patients and 44 healthy controls viewed 5 -s videos showing human faces gradually changing from neutral to fearful or happy expressions or from the neutral face of one poser to the neutral face of another. Recognition performance was determined separately by selfreport. Relative to prestimulus baseline, controls exhibited a $10-$ to $15-\mathrm{Hz}$ power increase prior to full recognition and a 10 - to $15-\mathrm{Hz}$ power decrease during the postrecognition phase. These results support recent proposals about the function of alpha-band oscillations in normal stimulus evaluation. The patients failed to show this sequence of alpha power increase and decrease and also showed low $10-$ to $15-\mathrm{Hz}$ power and high 10 - to $15-\mathrm{Hz}$ connectivity during the prestimulus baseline. In light of the proposal that a combination of alpha power increase and functional disconnection
\end{abstract}

T. G. Popov • B. S. Rockstroh • P. Popova • A. M. Carolus • G. A. Miller

Department of Psychology, University of Konstanz, Konstanz, Germany

G. A. Miller

Department of Psychology, University of California, Los Angeles, CA, USA

\section{B. S. Rockstroh $(\bowtie)$}

Department of Psychology, University of Konstanz, P.O. Box D23, D 78457 Konstanz, Germany

e mail: brigitte.rockstroh@uni konstanz.de facilitates information intake and processing, the finding of an abnormal association of low baseline alpha power and high connectivity in schizophrenia suggests a state of impaired readiness that fosters abnormal dynamics during facial affect recognition.

Keywords Schizophrenia $\cdot$ Facial affect recognition · Alpha oscillations $\cdot$ Emotion $\cdot$ Event processing $\cdot$ Functional connectivity $\cdot$ Neural networks

Schizophrenia patients exhibit altered affect processing in general, and impaired facial affect processing in particular (e.g., Delvecchio, Sugranyes, \& Frangou, 2013; Irani, Seligman, Kamath, Kohler, \& Gur, 2012; Johnstone et al., 2010; Kohler, Walker, Martin, Healey, \& Moberg, 2010; Kurtz \& Richardson, 2011; Sugranyes, Kyriakopoulos, Corrigall, Taylor, \& Frangou, 2011; Taylor et al., 2012). Impaired facial affect recognition markedly affects social function in schizophrenia (e.g., Hofer et al., 2009; Irani et al., 2012; Pinkham, Gur, \& Gur, 2007; see also Kurtz \& Richardon, 2011). The specificity of this deficit is less clear. It may depend on facial affect recognition and discrimination (Alfimova et al., 2009; Mandal, Pandey, \& Prasad, 1998; Mueser, Penn, Blanchard, \& Bellack, 1997; Pomarol-Clotet et al., 2010; Seyferth et al., 2009; Silver, Bilker, \& Goodman; Turetsky et al., 2007; Wölwer et al., 2012); it may be valencespecific, arising for specific facial emotions such as fear (e.g., Hall et al., 2008, and Leppänen et al., 2006, vs. Fiszdon \& Bell, 2009) or threat (e.g., Satterthwaite et al., 2010); or it may be a consequence of broader and more basic facial- or visualprocessing deficits (Chan, Li, Cheung, \& Gong, 2010; Kohler et al., 2010; Norton, McBain, Holt, Ongur, \& Chen, 2009; Pomarol-Clotet et al., 2010; Silver et al., 2009; Wynn, Lee, Horan, \& Green, 2008).

Identifying the deficit has prompted a number of hemodynamic and electrophysiological neuroimaging studies addressing 
the neural mechanisms associated with the behavioral deficits (see meta-analyses by, e.g., Delvecchio et al., 2013; Li, Chan, McAlonan, \& Gong, 2010; Sugranyes et al., 2011; Taylor et al., 2012; Taylor \& MacDonald, 2012). These studies have provided evidence of cortical (e.g., frontal, frontotemporal, and anterior cingulate) and subcortical (particularly amygdala) involvement in affect processing, facial affect recognition, and their dysfunction in schizophrenia. Understanding the dynamics of dysfunctional affect processing will also involve neural communication, including oscillatory brain activity and connectivity measures.

Distinct from face recognition per se, processing emotional expression in faces (as is common in social contexts) has been proposed to involve the recruitment of aspects of the neural and peripheral physiological events that occur when one experiences the emotion oneself (Lang, 1979; Niedenthal, 2007). Such recruitment of neural events when experiencing one's own emotions has been related to sensorimotor cortex activity (Adolphs, Damasio, Tranel, Cooper, \& Damasio, 2000; van der Gaag, Mindera, \& Keysers, 2007). Deficient recruitment in schizophrenia is suggested by diminished emotional contagion when schizophrenia patients are asked to produce matching or incongruous facial expressions (Falkenberg, Bartels, \& Wild, 2008). This deficit may contribute to reported deficits in theory of mind (e.g., Bora, Yucel, \& Pantelis, 2009; Frith \& Corcoran, 1996) and empathy (Besche-Richard, BourrinTisseron, Olivier, Cuervo-Lombard, \& Limosin, 2012) among schizophrenia patients. Taylor and MacDonald (2012) identified "embodied simulation of low-level mental state inference" (p. 73) as one of five promising research targets for understanding socio-emotional processing in schizophrenia.

Brain oscillations near the traditional 8- to $13-\mathrm{Hz}$ alpha band may be particularly suitable for tracking this neural recruitment. As was proposed by Jensen and Mazaheri (2010), the level of alpha oscillations reflects the excitatoryinhibitory states of neural ensembles, with event-related alpha power decreases (also called event related desynchronization, or ERD) reflecting excitatory, and alpha power increases (also called event related synchronization, or ERS) reflecting inhibitory, states (Haegens, Nacher, Luna, Romo, \& Jensen, 2011; Klimesch, 2012; Klimesch, Sauseng, \& Hanslmayr, 2007). Such alpha oscillations are related to long-range communication across networks and are thought to mediate information sampling and access to stored information (Klimesch, 2012). Indeed, alpha suppression (ERD) has been found when individuals perform, watch, or imagine actions in a social context (Pineda, 2005; Singh, Pineda, \& Cadenhead, 2011). Hence, alpha oscillation, specifically ERD near sensorimotor cortex, is linked to social information processing, which would include facial affect processing in many natural social contexts. Miller, Crocker, Spielberg, Infantolino, and Heller (2013) discussed how this view of alpha reflecting active mechanisms of information flow control contrasts with the traditional notion of alpha as reflecting an awake brain at rest.
In recent studies, researchers have begun to investigate relevant oscillatory dynamics in schizophrenia. Singh et al. (2011) found that stimuli showing moving hands or nomovement social interaction prompted less 8 - to $13-\mathrm{Hz}$ suppression (ERD) over sensorimotor cortex in first-episode schizophrenia patients than in controls. In healthy volunteers, Hars, Hars, Stam, and Calmets (2011) found greater 8- and 10$\mathrm{Hz}$ connectivity for sensorimotor cortex during the observation of biological motion. In addition, electroencephalographic (EEG) and magnetoencephalographic (MEG) findings of deviantly increased or decreased baseline alpha (Ikezawa et al., 2011; Nikulin, Jönsson, \& Brismar, 2012; Uhlhaas, Haenschel, Nikolic, \& Sincer, 2008; Uhlhaas \& Singer, 2010) suggest dysfunctional regulation of alpha oscillations in schizophrenia. Moreover, reduced alpha ERD has been reported in schizophrenia (although in nonemotional, auditory sensory gating [Popov et al., 2011] or oddball [Higashima et al., 2007; Koh et al., 2011]), and exaggerated alpha ERS has been found in working memory tasks (Bachmann et al., 2008). Finally, in addition to ERD and ERS within regions, the measurement of cross-time and cross-regional synchrony assesses abnormal oscillatory activity in schizophrenia. Nikulin et al. (2012) found correlations of alpha activity over time (reflecting long-range communication) to be reduced in schizophrenia patients. Hinkley et al. (2011) suggested that reduced resting-state coherence of alpha power between brain regions is a sign of functional disconnection in schizophrenia.

Trial-by-trial analysis of brain oscillatory activity offers further information about the dynamics of disturbed facial affect processing in schizophrenia. Moreover, the analysis of oscillatory dynamics within a trial facilitates the study of processing dynamically changing facial expressions (in contrast to static facial stimuli), which is closer to daily life. Garrido-Vasquez, Jessen, and Kotz (2011) summarized evidence that stimulus dynamics (e.g., videos vs. static faces) modify facial affect processing. The identification of affect in dynamically changing faces seems to be impaired in schizophrenia patients, whereas their performance benefits from nonfacial motion. For example, by comparing static and dynamic presentations of facial emotion, facial identity, and butterfly wings, Johnstone et al. (2010) found a specific deficit in the recognition of dynamic facial emotions that was not evident with static pictures.

In a study of healthy volunteers, Popov, Miller, Rockstroh, and Weisz (2013) examined the spatio-temporal dynamics of alpha activity during facial affect processing, using temporally dynamic stimuli to foster the investigation of the psychological and neural dynamics of face processing. Gradually morphing facial stimuli were used to analyze the process of affect recognition and cortical changes that support recognition. Oscillatory brain activity tracked recognition as faces changed from neutral to emotional (fearful or happy) expressions across 5 -s videos. Changes from neutral to emotional 
were associated with a 10 - to $15-\mathrm{Hz}$ power increase localized to bilateral sensorimotor areas preceding the time when identification of the facial emotional expression approached nearly $100 \%$ accuracy, followed by a power decrease. In the course of a trial, the 10- to $15-\mathrm{Hz}$ power increase was associated with decreased connectedness of the sensorimotor areas to other brain areas, including visual and fusiform face areas. The 10to $15-\mathrm{Hz}$ power decrease in the period following correct affect identification was associated with enhanced long-range connectedness of sensorimotor to other cortical areas. It was concluded that alpha oscillatory activity mediates a decoupling/coupling sequence that fosters facial affect recognition.

Event-related brain potentials (ERPs; e.g., Jung, Kim, Kim, Im, \& Lee, 2012; Lynn \& Salisbury, 2008), hemodynamic effects (e.g., Sato, Kochiyama, Yoshikawa, Naito, \& Matsumura, 2004), and event-related oscillatory activity (e.g., Ramos-Loyo, Gonzales-Garrido, Sanchez-Loyo, Medina, \& Basar-Eroglu, 2009) have been studied in schizophrenia patients employing static stimuli, and ERP and hemodynamic effects have been studied with dynamic faces in healthy participants, but not in schizophrenia patients (e.g., Fichtenholz, Hopfinger, Graham, Detwiler, \& LaBar, 2009; Mayes, Pipingas, Silberstein, \& Johnstone, 2009; Simon, Craig, Miltner, \& Rainville, 2006). Oscillatory activity in sensorimotor areas has been studied in schizophrenia patients, but not with emotional stimuli (Winograd-Gurvich, Fitzgerald, Georgiou-Karistianis, Millist, \& White, 2008). Altered connectivity in the neural networks relevant to facial affect processing in schizophrenia has been addressed in hemodynamic studies, though only with static stimuli (e.g., Leitman et al., 2008; Mukherjee et al., 2012) or emotional but nonfacial stimuli (Harvey et al., 2011). No study has combined the analysis of within-trial oscillatory activity during static and dynamic phases of facial affect recognition with within- and acrossregional coupling in schizophrenia patients.

Therefore, the present study used the design of Popov et al. (2013) to address the temporal specificity of oscillatory differences during the recognition process and to determine whether group differences are confined to pre- or postrecognition intervals, or even precede stimulus delivery. The primary hypotheses were that

1. Patients would correctly identify a fearful or happy expression later in a dynamically unfolding facial expression than would controls.

2. Patients would exhibit less modulation of alpha power (ERS and ERD) across the dynamic unfolding of a facial expression than would controls.

To evaluate the temporal and functional specificity of such findings, additional analyses were planned, in order to examine brain dynamics preceding the onset of changes in facial stimuli. The null hypotheses were that
3. Patients would not differ from controls in pretrial baseline alpha activity. Given the scarce evidence regarding a relationship between baseline and task-related alpha activity, we had no expectation of the extent to which baseline alpha activity would be related to oscillatory dynamics during the recognition period.

4. Group differences in oscillatory dynamics during the processing of unfolding emotional expressions would not be related to (group-specific) early event-related potentials in response to the onsets of the facial stimuli (in particular, the N170/M170).

\section{Method}

Participants

The study included 44 in-patients (SZ; 31 male, 13 female; mean age $32.0 \pm 9.4$ years) meeting an ICD-10 diagnosis of schizophrenia, and 44 healthy participants (HC; 24 male, 20 female; mean age $29.2 \pm 7.9$ years; the data from $n=30$ of the group were reported in Popov et al., 2013). The groups did not differ with respect to mean age $[t(86)=1.50, p=.13]$ or gender distribution $\left(\chi^{2}=2.4, p=.12\right)$. The $\mathrm{SZ}$ were recruited and diagnosed at the research unit of the regional Center for Psychiatry. Of the patients, 42 met an ICD diagnosis of paranoid-hallucinatory schizophrenia (code number F20.0), and two received diagnoses of schizoaffective (code number F25.0) or psychotic episode (F23.0). The inclusion criteria were normal intellectual function and no history of any neurological condition or disorder, including epilepsy or head trauma with loss of consciousness. The SZ participants were diagnosed by experienced senior psychiatrists or psychologists using the ICD-10 criteria. At the time of the assessment, the disease status was characterized by the Positive and Negative Syndrome Scale (PANSS: Kay, Fiszbein, \& Opler, 1987) symptom score (mean score $\pm S D$ : PANSS-P, $16.3 \pm 6.1$; PANSS-N, $20.2 \pm 6.1$; PANSS-G, $36.7 \pm 8.4)$ and global assessment of functioning ( $M \pm S D: 41.7 \pm 10.4)$. All patients were medicated, with a mean chlorpromazine equivalent of $722.6 \pm 441.4 \mathrm{mg} /$ day. The HC were screened with the Mini International Neuropsychiatric Interview (Ackenheil, StotzIngenlath, Dietz-Bauer, \& Vossen, 1999) in order to exclude any psychiatric or neurological disorder. All participants had normal or corrected-to-normal vision. Three SZ and five HC were left-handed, and three SZ and two HC were ambidextrous according to the Edinburgh Handedness Inventory (Oldfield, 1971).

Participants provided written informed consent prior to the experiment and received $€ 40$ at the end of the study. The study was approved by the ethics committee of the University of Konstanz. 
Materials and procedure

Pictures of 20 Caucasian male and 20 Caucasian female faces showing fearful, neutral, or happy expressions were selected from the Radboud Faces Database (Langner et al., 2010). Each of the 40 posers provided all three emotional expressions (fear, neutral, and happy), from which 120 motion videos were created. For each poser, two videos showed a transition from a neutral face to a fearful expression (NF) or a happy expression (NH). In the third video, the facial features (mouth, nose, or eye) of a neutral face of one model were altered toward the neutral face of another model of the same gender (NN). Thus, the feature change required the recognition of a change in poser identity rather than recognition of a change in emotional expression. This condition controlled for the effects of recognizing a change of invariant facial aspects without emotional involvement. Fivesecond videos were created in-house using the face morphing software Fantamorph (www.fantamorph.com/).

During MEG recording, the participants were instructed to passively view the video sequences. During the first second of each 5-s trial, the video presented a static image of the initial (neutral) expression. The images gradually morphed toward the target facial expression (the same model with either a fearful or happy expression or a different model with a neutral expression) at a rate of 15 frames per second across $3 \mathrm{~s}$, such that $33 \%$ of the final expression was reached at the end of the second second and $100 \%$ at the end of the fourth second (see Fig. 1). During the fifth second, the video presented a static image of the final expression. A gray mask covered the hair, ears, and neck, so that for all stimuli only the face was seen, in order to keep figure-ground contrasts constant.

Across the 120 videos, $40 \mathrm{NF}, 40 \mathrm{NN}$, and $40 \mathrm{NH}$ morphs were presented in pseudorandom order, separated by a 5-s $( \pm 1 \mathrm{~s})$ jittered offset-to-onset intertrial interval (ITI), during which a white fixation cross appeared in the center of a black screen. The videos were presented on a screen about $50 \mathrm{~cm}$ distant from the participant's eyes during MEG recording. Recording lasted approximately $18 \mathrm{~min}$.

Each participant's recognition performance was assessed by self-report ratings of static video images in a subsequent session. (Self-report data were available for 36 patients and 30 controls.) For these ratings, seven frames were exported from ten videos of five male and five female actors, representing seven levels of transition (neutral/emotional, as percentages: $80 / 20,70 / 30,60 / 40,50 / 50,40 / 60,30 / 70$, and 20/80). Thus, 140 pictures were used in the rating sessions. A picture was presented randomly on a computer screen for $50 \mathrm{~ms}$ after a $2-\mathrm{s}$ fixation-cross (baseline) period. Drawings of three manikins then appeared. Their eyes and mouths were formed to indicate fearful, neutral, or happy expressions. The (German) words for "fearful," "neutral," and "happy" were printed underneath the faces. Participants were instructed to move the cursor to the manikin that reflected the emotion of the face just presented and to confirm their decision by a left mouse click. A subsequent left mouse click brought the next picture. Performance scores were calculated by dividing emotional by neutral accuracy for each morph level and poser. Chance performance was $33 \%$.

\section{Data acquisition and analysis}

MEG was recorded with a 148-channel whole-cortex magnetometer (MAGNES 2500 WH, 4-D Neuroimaging, San Diego, USA) in a magnetically shielded room while participants lay on their back. Prior to each session, each participant's nasion, inion, $\mathrm{Cz}$, left and right ear canal, and head shape were digitized with a Polhemus 3Space Fasttrack. Participants were instructed to passively watch the videos and to avoid body movements. The continuous MEG time series was recorded with a sampling rate of $678.17 \mathrm{~Hz}$ and a 0.1 - to $200-\mathrm{Hz}$ band-pass filter. Trials consisted of epochs from $3 \mathrm{~s}$ before to $7 \mathrm{~s}$ after the onset of each video. Prior to correcting for heart and eye-blink artifacts by means of independent component analysis (ICA), trials containing movement artifacts and SQUID jumps were rejected on the basis of visual inspection. Groups did not differ in the numbers of trials retained in any of the three conditions, nor did the conditions differ in this respect (for HC: NF, $38.3 \pm 2.0$; $\mathrm{NN}, 38.2 \pm 2.1$; NH, $38.9 \pm 1.4$; for $\mathrm{SZ}: \mathrm{NF}, 37.3 \pm 3.0$; NN, $37.7 \pm 2.0 ; \mathrm{NH}, 37.8 \pm 2.4)$.

Planar gradient calculation The MEG data were transformed into a planar gradient configuration for each sensor using signals from neighboring sensors (Bastiaansen \& Knosche, 2000). This procedure generally emphasizes activity at sensors located above the actual sources, simplifying the interpretation of sensor-level data (Hämäläinen, Hari, Ilmoniemi, Knuutila, \& Lounasmaa, 1993).

Frequency analysis Spectral analysis was computed for each trial using an adaptive sliding time window of five cycles length $(\Delta \mathrm{t}=5 / f)$ multiplied by a Hanning taper. Resulting power estimates were averaged over trials within condition. Time-frequency representations of power were calculated as change from prevideo (prestimulus) baseline in each condition (NN, NH, NF). Then the time-frequency representations of power for the $\mathrm{NN}$ averages were subtracted from both the $\mathrm{NF}$ and the $\mathrm{NH}$ averages, producing $\mathrm{NF}-\mathrm{NN}$ and $\mathrm{NH}-\mathrm{NN}$ differences for each participant.

Source analysis Source reconstruction was based on individual structural MRIs and on an affine transformation of an MNI-template brain (Montreal Neurological Institute (MNI), Montreal, Canada www.bic.mni.mcgill.ca/brainweb) to the participant's digitized individual head shape $(N=5 \mathrm{HC}, N=$ 23 SZ; see also Keil, Weisz, Paul-Jordanov, \& Wienbruch, 


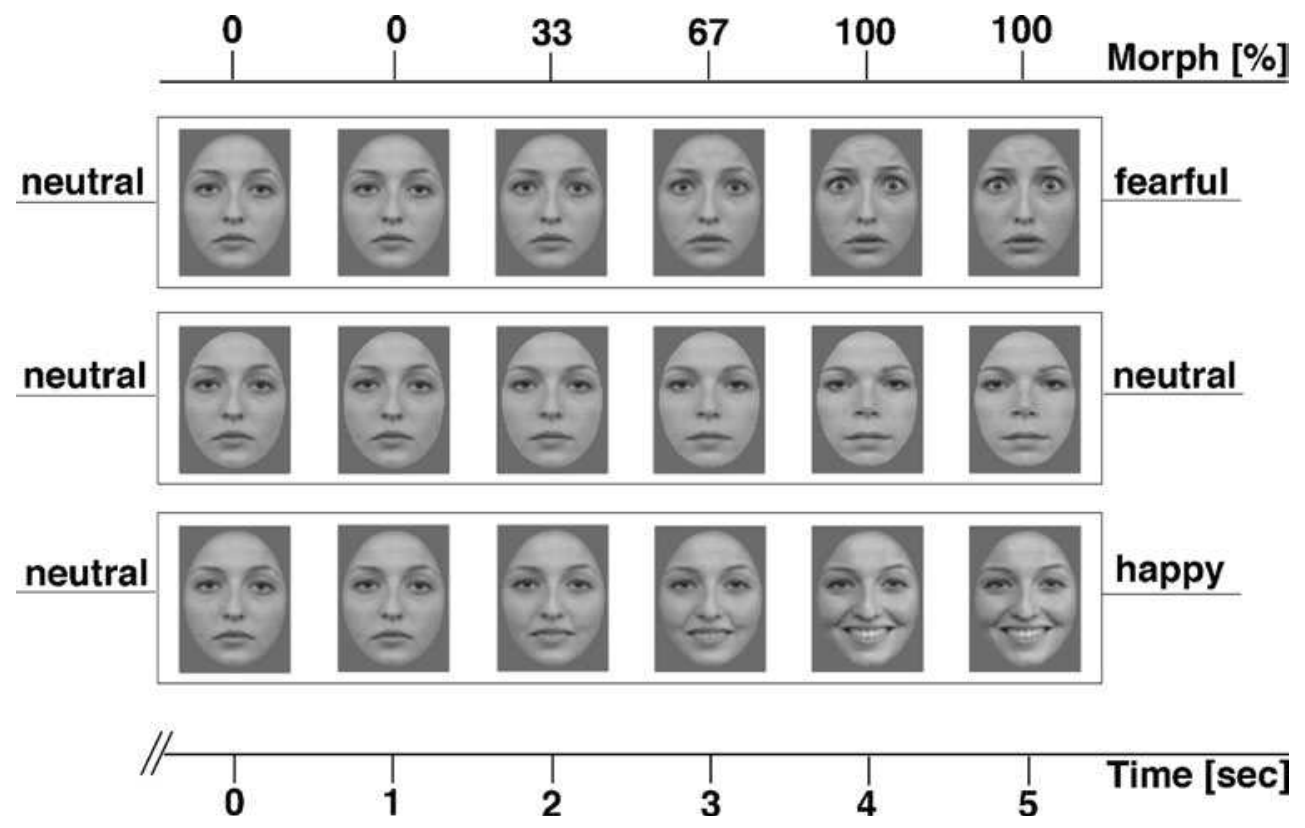

Fig. 1 Examples of morphed pictures used for videos during magnetoen cephalography (MEG) and the subsequent rating task. The $x$ axis above the faces indicates the percentage of emotional expression in the particular frame. The axis below the faces indicates time during the video presented during the MEG session. The top row of faces illustrates the transition from a neutral to a fearful expression (NF), the middle row the transition from one poser to another (both neutral, $\mathrm{NN}$ ), and the bottom row the transition from a neutral to a happy expression (NH). From "Modulation of Alpha Power and Functional Connectivity During Facial Affect Recognition," by T. Popov, G. A. Miller, B. Rockstroh, and N. Weisz, 2013, Journal of Neuroscience, 33, pp. 6018 6026. Copyright 2013 by the Society for Neuroscience. Adapted with permission
2010). A frequency-domain adaptive spatial filtering algorithm (dynamic imaging of coherent sources; DICS) served to estimate the sources of activity that contributed to the effects at the sensor level (Gross et al., 2001; for details, see the supplementary materials). Calculated $z$ values represented the normalized differences of source estimates between prestimulus baseline and recognition-related time windows. Source estimates were interpolated onto the individual anatomical images and subsequently normalized to a standard Montreal Neurological Institute (MNI) brain in order to calculate group statistics and for illustrative purposes. Group differences were calculated using independent-sample $t$ tests. If not indicated otherwise, all reported comparisons and thresholds in the statistical images used an alpha level of $p<.05$.

Functional connectivity analysis Brain connectivity analysis was done via graph-theoretic metrics (Bullmore \& Sporns, 2009; for details, see the supplementary materials and Popov et al., 2013). The functional connectivity measure was the phase synchrony of pairs of voxels (Lachaux, Rodriguez, Martinerie, \& Varela, 1999), similar to the procedures described in Keil, Mueller, Ihssen, and Weisz (2012). Phase synchrony was computed for time and frequency windows of interest that were derived from the sensor-level analysis, for each voxel relative to all other voxels in the entire cortical volume. A relationship between two voxels was considered meaningful if the probability under the null hypothesis that the phase differences are drawn from a uniform distribution was $0.1 \%$ (Rayleigh test; Fisher, 1993). Node degrees were also calculated using thresholds of both $5 \%$ and $1 \%$. Since the outcomes were virtually identical, only the data thresholded at $0.1 \%$ are reported.

Network analysis The functional connectivity analysis yielded an adjacency matrix from which the graph-theoretical metric "node degree" could be calculated using the Brain Connectivity Toolbox (www.brain-connectivity-toolbox.net; Rubinov $\&$ Sporns, 2010). This measure is a global index of the connectedness of a brain region, identifying brain regions generally coupled or decoupled during the encoding of facial affect. Finally, the task-related degree of connectivity was expressed relative to the connectivity degree of the baseline period ([task degree - baseline degree]/baseline degree), in which positive values indicated increased connectivity to all other brain regions, and negative values decreased connectivity (decoupling).

Statistical analysis Self-reported recognition performance scores (see above) were compared in a repeated measures analysis of variance with the between-subjects factor Group (SZ, HC), the within-subjects factor Transition ( $30 \%, 40 \%$, $50 \%, 60 \%$, and $70 \%$ morphed emotion), and the withinsubjects factor Emotion (transition from neutral to fearful or to happy expression). Significant main effects and interactions were explored with post-hoc $t$ tests. (Because the two extreme 
levels of transition [20/80 and 80/20] produced $>90 \%$ correct recognition of the facial expression in all participants, they were not considered in the analysis of recognition accuracy.)

For the MEG data, time-frequency windows of significant differences between conditions in alpha activity were defined for each participant via cluster-based, independent-sample $t$ tests with Monte Carlo randomization that effectively controlled for multiple comparisons (Maris \& Oostenveld, 2007). The Monte Carlo estimate describes the comparison of randomized test statistics with the observed test statistic and identifies sensor clusters with significant group differences on the sensor level, as well as sources with significant group differences on the source level. Pairwise differences between conditions (NN-NF, NN-NH, and NF-NH) in sensor clusters were accepted as reliable when they were below the $5 \%$ level, with the test statistic having been defined as the sum of the $t$ statistics of the sensors within the respective cluster. Normalized power changes ([activity - baseline] / baseline) were computed separately for the three conditions at each sensor. The baseline period was defined as $3 \mathrm{~s}$ before face onset. This cluster-based, independent-sample $t$ test statistic was also used to define the time windows of significant group differences in event-related magnetic field strength (ERF) during the first $500 \mathrm{~ms}$ following video onset and to define group differences in alpha power during the 3 -s prevideo baseline period. In addition, an effect size $d$ was calculated following the procedures described by Rosnow and Rosenthal (2003), with $d=t / \sqrt{ } d f$.

Offline treatment of the MEG signals was accomplished primarily with the MATLAB-based, open-source signalprocessing toolbox Fieldtrip (Oostenveld, Fries, Maris, \& Schoffelen, 2011), complemented by in-house MATLAB functions.

\section{Results}

\section{Recognition performance}

Figure 2 illustrates recognition performance based on ratings done following the MEG measurement. Recognition accuracy improved during the transition, from chance to nearly $100 \%$ correct recognition for faces expressing more than $70 \%$ of the respective emotion. For the 50\%-70\% transition level, the number of participants who reached the level of $90 \%-100 \%$ correct identification increased from $70 \%$ to $93 \%$ (neutral to fearful) and $77 \%$ or $93 \%$ (neutral to happy) in $\mathrm{HC}$, whereas the respective increase for SZ was from $47 \%$ to $81 \%$ (neutral to fearful) or $39 \%$ to $83 \%$ (neutral to happy). A general increase in recognition accuracy with increasing proportions of morphed emotion, which was more rapid for the transition to happy than to fearful, was confirmed by an effect of transition $[F(4,256)=495.16, p<.001$, Huynh-Feldt $\varepsilon=.65]$ and an Emotion $\times$ Transition interaction $[F(4,256)=$ $4.50, p<.003, \varepsilon=.82]$.

Figure 2 suggests that SZ were less accurate in identifying fearful expressions near $50 \%-60 \%$ transition and less accurate in identifying happy expressions near $40 \%-50 \%$ than were HC. The SZ group's recognition performance was worse overall $[F(1,64)=5.43, p=.02]$. Although the Group $\times$ Transition $(p=.18)$ and Group $\times$ Transition $\times$ Emotion $(p=.10)$ interaction effects were not significant, simple-effects tests were done in order to ensure that the selection of analysis windows distinguishing pre- and postrecognition periods minimized any possible confound with group differences in recognition performance. These $t$ tests indicated group differences in the recognition of fearful expressions at $50 \%[t(64)=2.10, p=.04]$ and $60 \%[t(64)=2.60, p=.01]$, in the recognition of happy expressions at $40 \%[t(64)=2.47, p=.02]$ and $50 \%[t(64)=$ $2.41, p=.02]$, and not at earlier or later morph stages.

\section{Alpha activity related to recognition processes}

Analysis epochs during the course of recognition were determined from performance data in the following way: As is evident from Fig. 3, changes in alpha activity started at the time point at which the transition from a neutral to an emotional face began. Given that the first second of the video presented a static neutral face, this transition period started $1 \mathrm{~s}$ after video onset. Subsequently, alpha power changes continued until the stage at which $>80 \%$ identification accuracy was achieved for faces reaching $70 \%$ of the morph transition, approximately $3 \mathrm{~s}$ after video onset. Therefore, the interval of $1-3 \mathrm{~s}$ after stimulus onset was scored to reflect the recognition interval (achieving correct facial affect identification). For analyses, this interval was defined as the "prerecognition period." After $70 \%$ of the morph transition, with performance that was nearly perfect and similar for the two groups, the period of 3.5-4.5 s after stimulus onset was analyzed as the "postrecognition period," in order to distinguish and evaluate brain activity before and after a clear stage of correct facial affect identification (i.e., recognition).

Alpha activity during the prerecognition period

Figure 3a illustrates oscillatory activity prior to onset of and during the 5-s video. In $\mathrm{HC}$, a prominent 10 - to $15-\mathrm{Hz}$ power increase from prevideo baseline is evident once face morphing began at $1 \mathrm{~s}$ after video onset, lasting until about $3 \mathrm{~s}$, when faces had reached $67 \%$ of the eventual emotion. This alpha power increase was followed by a return to near baseline in alpha power during the final $2 \mathrm{~s}$ of the video. Figure 3 a suggests no such overall sequence in SZ, with little change from baseline throughout the video.

Panels B-D of Fig. 3 illustrate group differences in alpha power and connectivity during the 1 - to 3 -s prerecognition 

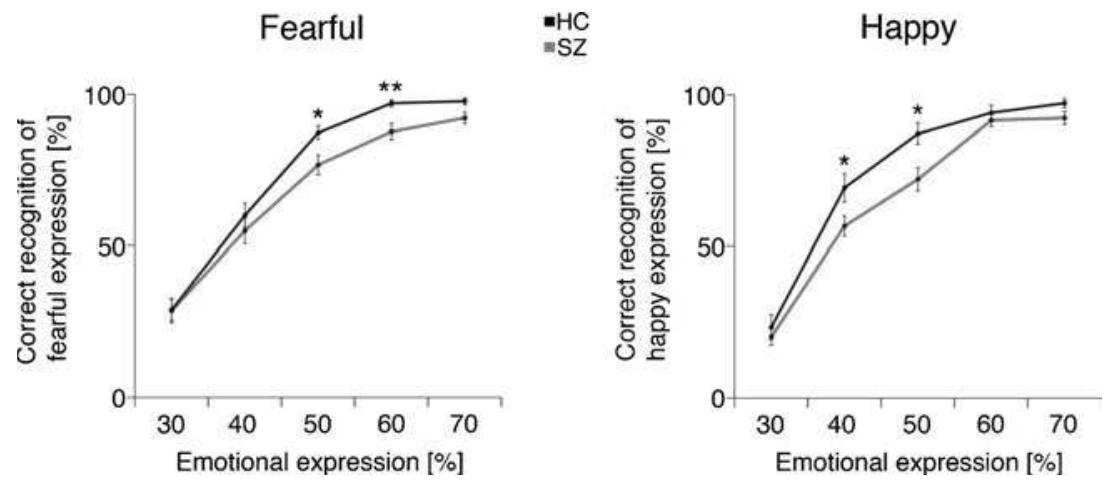

Fig. 2 Mean percentages of correct decisions in the rating session as a function of the proportion of transitions from neutral to emotional expressions, spanning the portion of the morphing from $30 \%$ to $70 \%$. Pairwise group differences are indicated by asterisks $\left({ }^{*} p<.05,{ }^{* * *} p<.01\right)$

period. Figure $3 \mathrm{~b}$ illustrates that $\mathrm{SZ}$ displayed significantly less of an alpha power increase than did $\mathrm{HC}$ at central sensor clusters: primarily left-hemisphere central for the fearful condition and more bilateral central for the happy condition. ${ }^{1}$

Source reconstruction (Fig. 3c) similarly shows significantly less alpha power increase during the prerecognition period in SZ in and near sensorimotor areas for the neutralto-happy transition, whereas the neutral-to-fearful transition prompted similar alpha power increases in bilateral sensorimotor areas in both groups. Figure $3 \mathrm{~d}$ illustrates functional connectivity patterns during the prerecognition period. During the prerecognition period for a subset of the present $\mathrm{HC}$ group, Popov et al. (2013) reported increased functional connectivity within sensorimotor areas, together with decoupling from other brain areas. This pattern of early power increase and interregional connectivity decrease was interpreted as reduced information flow from other regions, thus facilitating communication within sensorimotor cortex. As is evident in Fig. 3d, the global index of network connectivity showed significantly less connectivity of central regions with all other regions and significantly more connectivity of sensory and left- and right-frontal brain regions with all other regions in SZ than in $\mathrm{HC}$.

\footnotetext{
${ }^{1}$ Comparison of the topographies of the group differences of condition effects in Fig. 3b with the condition effects displayed separately for each group in Supplementary Fig. 1 indicates that SZ exhibited weaker bilateral generators than did $\mathrm{HC}$ for all transitions (NF, NN, and $\mathrm{NH}$ ). For HC, the topography of the transition effects suggests a tendency for higher right than left hemisphere activity across all conditions, includ ing NN. Contrasting these condition effects by groups (bottom row of Supplementary Fig. 1) resulted in topographical group differences for the NN morph. Thus, subtracting this NN NN data from the NN NF and NN NH data produced slightly different topographies. Because of the similarity of the condition effects in both groups and the similarity of the topographies within each group, as are shown in Supplementary Fig. 1, the topographical group differences in Fig. 3b generally reflect these contrasts rather than indicating group specific affect processing.
}

Alpha activity during the postrecognition period

Figure 4 presents results for the postrecognition time window. Figure 4a suggests less of an alpha power decrease in SZ than in $\mathrm{HC}$ for transitions to fearful or happy expressions. Figure $4 \mathrm{~b}$ confirms the significant group differences in sensor space, primarily over midline and right-posterior regions. Source reconstruction (Fig. 4c) shows significantly less alpha power decrease in SZ than in $\mathrm{HC}$ in frontal and central regions, and Fig. 4d illustrates significantly lower interregional connectivity of central regions in SZ than in $\mathrm{HC}$, similar to that described for the prerecognition period in Fig. 3d.

In summary, SZ did not show the sequence of alpha power increase/recovery and connectivity decrease/recovery in sensorimotor cortex found for HC. Before interpreting this as showing a cortical abnormality specific to face processing, activity preceding the recognition interval should be examined.

Alpha activity and connectivity prior to dynamic face stimuli

Given the group differences in oscillatory activity during the recognition task, it would be important to evaluate whether they were confined to the period of task performance or arose earlier. Figure 5 illustrates the time course of the ERF during the first $500 \mathrm{~ms}$ of the $1-\mathrm{s}$ static face that preceded the morphing process. Figure 5 is confined to activity localized to the right fusiform gyrus, a key area in early face processing. As we expected, a clear M170 component is apparent. Importantly, SZ and HC did not differ in M170 source strength $[t(86)=-1.62, p=.11]$ (see Supplementary Fig. 2). In fact, the SZ mean was slightly higher than the HC mean, so the lack of a group difference was not due to inadequate statistical power to find a smaller M170 in SZ than in HC. Thus, patients' early cortical face-specific registration was intact.

Although SZ produced a normal M170, suggesting normal registration of faces, Fig. 6 illustrates the presence of significantly less alpha power during the 3 -s prevideo baseline (averaged across frontal-central sensors and trials) in SZ 
a
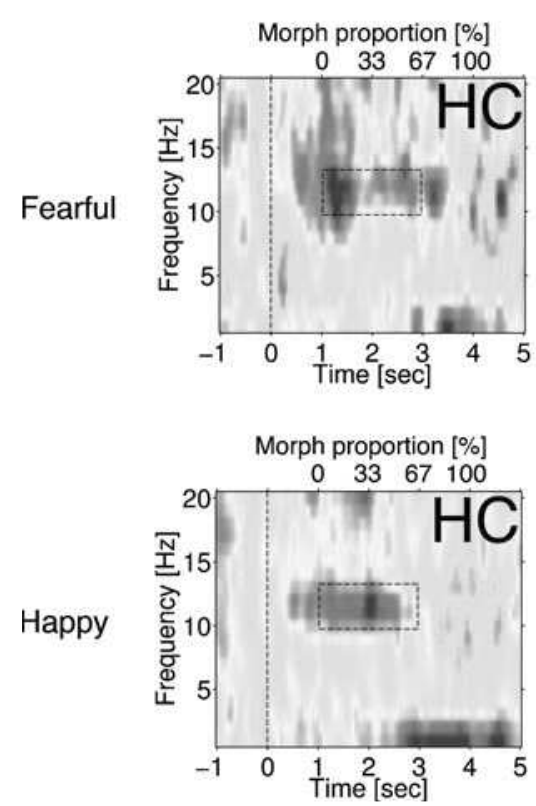

Happy
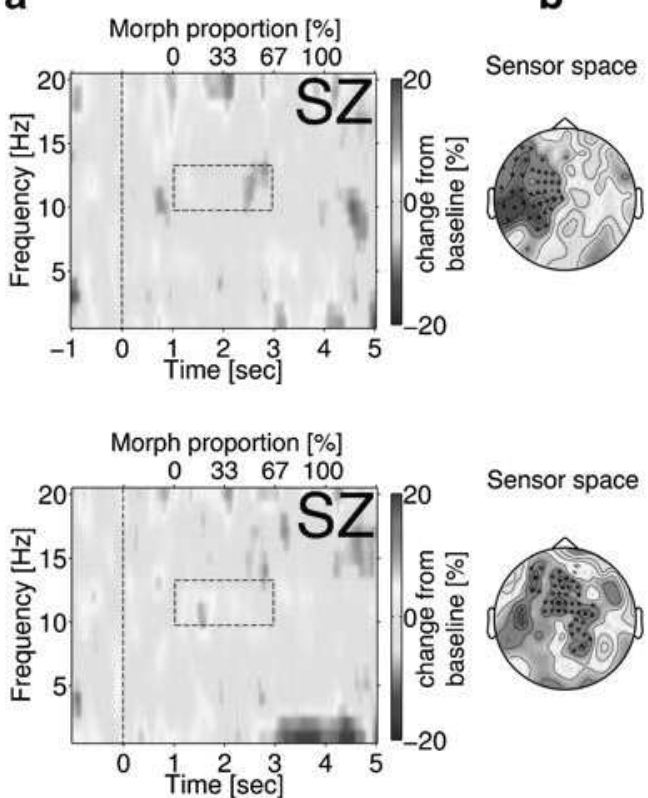

C

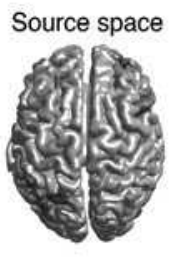

d

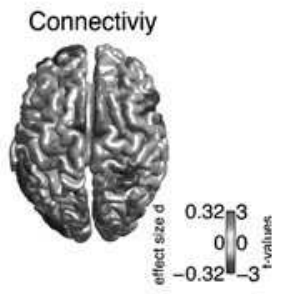

Fig. 3 a Time frequency representations of power as a function of transi tion from neutral to fearful or happy expressions, each minus the transition from neutral to neutral, averaged across the sensors marked as black dots in column $b$. Warm colors indicate larger increases in power change from prestimulus baseline for emotional expressions. Video onset was at $0 \mathrm{~s}$, face morph onset at $1 \mathrm{~s}$, face morph offset at $4 \mathrm{~s}$, and video offset at $5 \mathrm{~s}$. The dashed rectangles mark the 1 to $3 \mathrm{~s}$ time window scored as the prerecognition period. b Group differences in scalp topography of 10 to

than in HC (Fig. 6b). Source reconstruction identified significant group differences in frontal, premotor, and sensorimotor areas (Fig. 6c). Finally, global functional connectivity showed significantly higher connectedness of these areas in SZ than in HC (Fig. 6d).

\section{Discussion}

The present study, designed to evaluate facial affect recognition in dynamically unfolding emotional expressions, found group differences in overt performance, oscillatory activity, and connectivity. The time course of HC participants reporting successful identification of morphing emotional expressions indicated that the task was effective. SZ participants took slightly but significantly longer to do so. Importantly, SZ failed to show the sequence of alpha power increase/recovery and connectivity decrease/recovery in sensorimotor cortex that the task evoked in HC. This was not due to a generalized deficit, but prestimulus baseline activity may have contributed to the SZ group's judgment impairment. Prior to face onset, sensorimotor cortex in SZ manifested lower alpha power and higher connectivity with the rest of cortex. This apparently led, upon stimulus presentation, to normal structural face registration (evidenced by
$15 \mathrm{~Hz}$ activity $13 \mathrm{~s}$ after stimulus onset, expressed as $t$ values, with warm colors indicating more activity in HC than in SZ. Black circles indicate the sensors belonging to a significant cluster. c Group differences in source reconstructions of the 10 to $15 \mathrm{~Hz}$ activity modulation associated with the effects shown in column $b$. Warm colors indicate stronger sources in HC than in SZ. d Group differences in global connectivity associated with the effects shown in column $b$. Warm colors indicate regions of higher connectivity in $\mathrm{HC}$ than in SZ. Color bars at the far right are for columns b, c, and d

normal M170) but a diminished ability to engage in the evaluation of dynamic facial emotion.

In line with the findings of Popov et al. (2013) on a subset of the present $\mathrm{HC}$ sample, the full $\mathrm{HC}$ sample exhibited a sequence of increased alpha power in sensorimotor cortex and reduced connectivity of this region with other regions during the prerecognition period, followed by alpha power decline and connectivity restoration during the postrecognition period. The role of alpha oscillations in perception and cognition has been characterized as "gating by inhibition" (Hanslmayr, Gross, Klimesch, \& Shapiro, 2011; Jensen, Bonneford, \& Van Rullen, 2012; Jensen \& Mazaheri, 2010; Klimesch, 2012; Klimesch et al., 2007; Miller et al., 2013). According to this view, synchronized alpha oscillations in local neural ensembles reflect the inhibitory state of these ensembles. Such local inhibition of input effectively controls information flow within and across networks.

In this framework, for $\mathrm{HC}$ the association of local alpha power increase and disconnection from other brain regions reflects a state of readiness for local information processing. The present results provide clear support for this view, further undermining the long-accepted assumption that alpha is merely a reflection of an awake but essentially idling brain. The present results also support the proposal that subthreshold efference can be a key element of processing emotional 
a
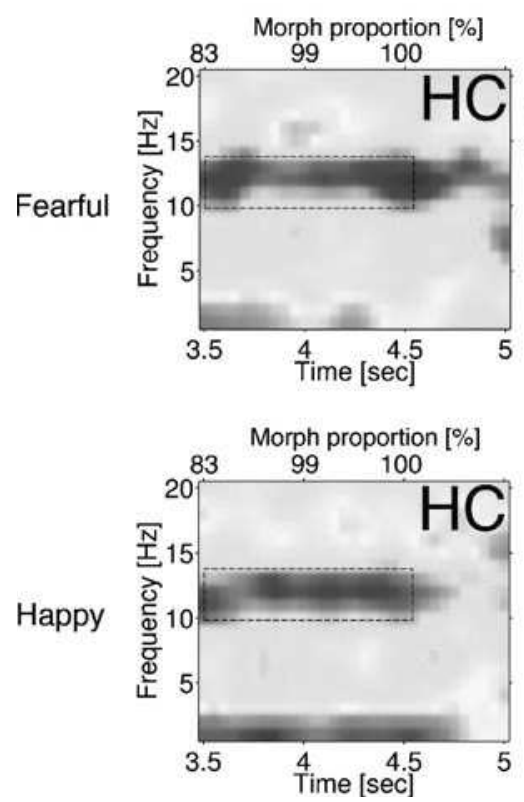
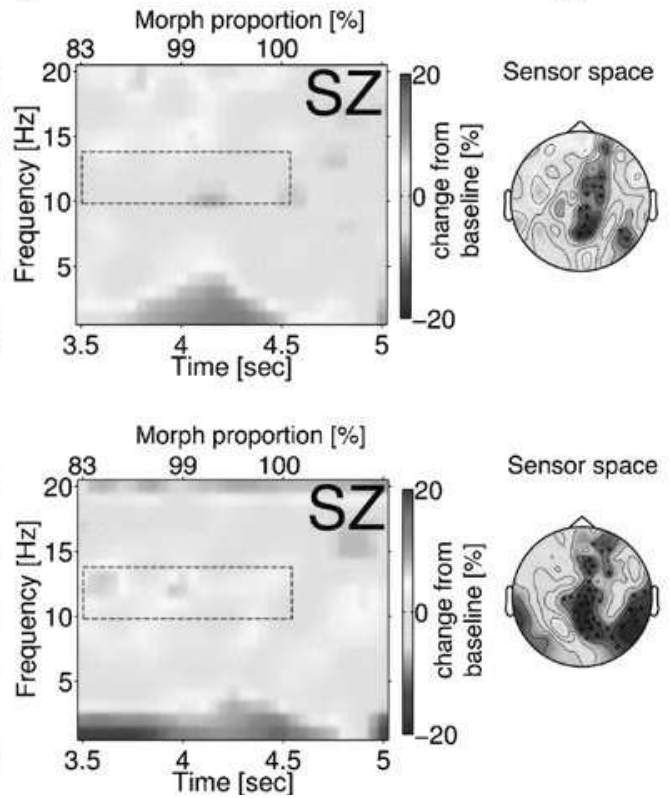

C
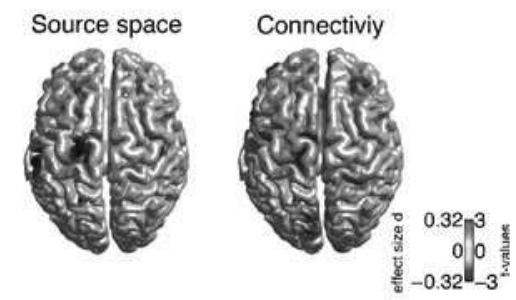

d

Fig. 4 a Time frequency representations of power as a function of transition from neutral to fearful or happy expressions, averaged across the sensors marked as black circles in column b. Cold colors indicate greater decreases in power change from prestimulus baseline for emo tional expressions. The dashed rectangles mark the 3.5 to $4.5 \mathrm{~s}$ time window scored as the postrecognition period. b Group differences in scalp topography of 10 to $15 \mathrm{~Hz}$ activity $3.54 .5 \mathrm{~s}$ after stimulus onset,

afference (Lang, 1979; Niedenthal, 2007; see also Iacoboni \& Dapretto, 2006).

In comparison to $\mathrm{HC}$, during the 1- to 3-s prerecognition period, SZ failed to show high alpha power and low connectivity. The static onset at the beginning of the trial prompted a normal M170 response, indicating registration of the face in the fusiform gyrus of the visual system, which is evidence against a generalized deficit, such as failure to attend to the video stream or to recognize the stimulus as a face. The subsequent dynamic processing failure presumably led to their delayed judgment about the face emotion. Reduced N170 has been reported in SZ during tasks that involved the comparison

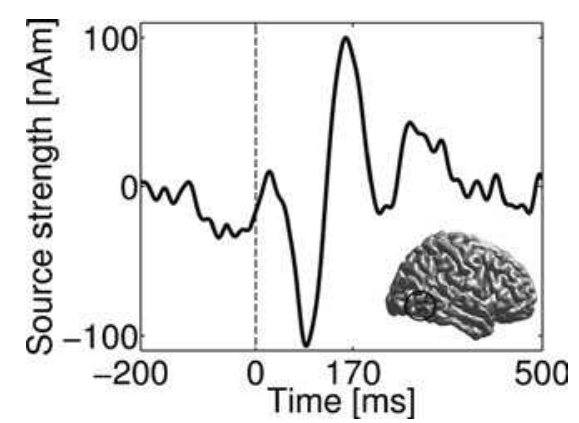

Fig. 5 Time course of neural source strength in and near the fusiform gyrus, indicated in the inset, for $500 \mathrm{~ms}$ following onset at $0 \mathrm{~ms}$ of a static neutral face that preceded the morphing process, relative to the $200 \mathrm{~ms}$ prestimulus baseline, averaged across all participants expressed as $t$ values, with warm colors indicating more activity in HC than in SZ. Black circles indicate sensors belonging to a significant cluster. c Group differences in source reconstructions of the 10 to 15 $\mathrm{Hz}$ activity modulation associated with the effects shown in column b. d Group differences in global connectivity associated with the effects shown in column $\mathrm{b}$. Warm colors indicate regions of higher connectivity in $\mathrm{HC}$ than in SZ. Color bars at the far right are for columns b, c, and d

of facial affect and facial feature elements or faces and objects (e.g., Turetsky et al., 2007; Wölwer et al., 2012; Wynn, Jahshan, Altshuler, Glahn, \& Green, 2013). Other studies have reported normal N170 in SZ (e.g., Wynn et al., 2008) or abnormal topographies in SZ (larger over occipital but smaller over frontal regions; Ramos-Loyo et al., 2009). Current source density analysis of MEG data from SZ (Streit et al., 2001) found weaker activity over left inferior parietal and right occipital regions in an early time window (100-240 ms, including M170), and in right fusiform gyrus in a somewhat later time window (160-300 ms). The presentation here of a 1s static neutral face, with no task required (prior to morphing), need not have prompted active comparative face evaluation. This may account for the lack of group differences in M170.

The SZ group's dynamic processing failure may result from their abnormally high interregional alpha connectivity prior to face onset. If the preparatory state preceding stimulus presentation and task performance is abnormal, manifest in both low alpha power and high connectivity, subsequent processing may be compromised. Thus, SZ undertook the task in a brain state poorly prepared for dynamic emotional face processing.

Connectedness among neural networks involved in affect processing in SZ has also been studied using fMRI. For fearful face identification, in particular, Leitman et al. (2008) found fewer interregional correlations between amygdala and frontal (medial, inferior, and prefrontal) regions in SZ than in $\mathrm{HC}$, but 
a

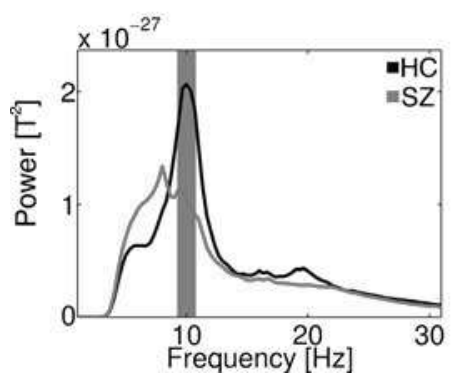

Fig. 6 a Average power for the significant clusters of sensors marked as black circles in column $\mathrm{b}$ during the $3 \mathrm{~s}$ baseline preceding video onset. The gray bar indexes the frequency range of significant group differences. b Scalp topography of group differences (expressed as $t$ values) in the differentiating frequency range (gray bar in column a). Black circles indicate sensors belonging to the significant cluster, and warm colors

higher correlations between occipital (lingual gyrus) and superior temporal gyrus in SZ than in HC. Mukherjee et al. (2012) reported lower connectivity between amygdala and parietal regions in an implicit facial affect recognition task, and Harvey et al. (2011) reported lower occipital to parietal and prefrontal coupling in SZ than in $\mathrm{HC}$, even in nonemotional visual perception tasks.

The low connectivity in these fMRI studies of SZ compares with a more nuanced picture in the present data. MEG allowed for the distinction of time courses across the baseline, prerecognition, and postrecognition periods, during which connectivity varied as a function of region and time. Evidence from different neuroimaging methods has indicated that disturbed neural coupling contributes to or is a manifestation of dysfunctional processing in SZ, but additional work is needed to map the role of these phenomena.

The present hypotheses about the role of alpha oscillatory activity in the processing of facial expressions were developed from evidence highlighting the role of sensorimotor areas in facial affect recognition (e.g., Adolphs et al., 2000; Pitcher, Garrido, Walsh, \& Duchaine, 2008), the significance of alpha oscillations for perception and cognition (Hanslmayr et al., 2011; Jensen et al., 2012; Klimesch, 2012; Klimesch et al., 2007), and the suppression of alpha (mu) activity over central EEG electrodes during observation of social interaction (Singh et al., 2011; Pineda \& Hecht, 2009). Considering the functional role of alpha oscillations in inhibition and timing of communication between neuronal networks (Klimesch, 2012), the present results suggest that patients undertook the task of facial affect recognition in a state of impaired inhibitory control and transregional communication.

The present results do not challenge evidence from hemodynamic and electrophysiological neuroimaging about several brain regions involved in facial affect recognition. The dysfunctional modulation of alpha oscillations prior to seeing facial stimuli and during the processing of dynamically unfolding emotional expression may well be a mechanism
C

Difference $\mathrm{HC}$ vs. SZ
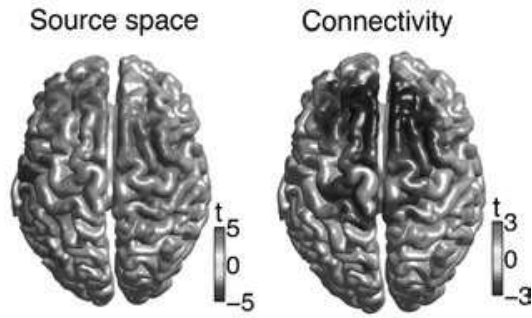

indicate more power in $\mathrm{HC}$ than in SZ. c Source reconstruction of alpha power group differences during baseline. Warm colors indicate voxels of higher baseline alpha power in HC than in SZ. d Group differences in connectivity for baseline alpha power, expressed as $t$ values. Bilateral regions in cool colors show higher connectivity in SZ than in $\mathrm{HC}$

of or additive to abnormal subcortical, frontal, and cingulate activity and their interaction in face recognition (Behrmann \& Plaut, 2013) and affect processing (Delvecchio et al., 2013; Taylor et al., 2012), which has been related to the deficient recruitment or "substantial limitation in activation throughout a ventral temporal-basal ganglia-prefrontal cortex 'social brain' system" (Li et al., 2010, p. 1029; see also Li et al., 2012). The present results point to an element specifically contributing to facial expression processing within this social brain system. Moreover, the unfolding of affect expression in a face is inseparably linked to biological motion (hand, face, or body), detection of which has been found to activate superior temporal sulcus (STS; e.g., Hars et al., 2011; Thompson, Hardee, Panayiotou, Crewther, \& Puce, 2007), even in tasks requiring emotion processing like theory of mind (Frith \& Frith, 1999; Krakowki, Ross, Snyder, Sehatpour, Kelly, \& Foxe, 2011; Sugranyes et al., 2011). No study seems to have examined biological motion and its cortical correlates specifically in SZ. The fact that the present results did not include group differences in STS alpha modulation indicates either that the present design did not activate STS differentially (biological motion being similarly involved in all conditions) or that SZ are not significantly impaired in biological motion processing, at least regarding facial motion. A future study comparing dynamically unfolding facial expressions (as in the present design) and nonfacial, nonemotional biological movement (hand or body) might clarify the specificity of this impairment in SZ.

Limitations of the present study can be noted. Recruitment of aspects of the central and peripheral physiology of one's own emotional efference (Lang, 1979) has been proposed as a prerequisite for the perception of emotion in others, particularly in natural social contexts (Niedenthal, 2007). However, the present design involved only facial stimuli and facial affect expression, which may have prompted involvement of the sensorimotor face area, regardless of emotion features. Thus, the specificity to emotional 
face stimuli of task-induced alpha power and alpha-mediated connectivity warrants evaluation by comparison with other perceptual-cognitive tasks.

The impact of task instruction on dysfunctional modulation of alpha oscillations remains to be examined through explicit comparison. The present facial affect recognition design did not require an explicit response during the MEG measurement but also did not resemble typical implicit task designs, in which key stimulus features are not relevant to the instructed task. Performance deficits and abnormal ERPs in SZ have been reported for explicit emotion identification or matching tasks (see the review by Garrido-Vasquez et al., 2011), whereas results on implicit tasks like affective priming are less consistent (Garrido-Vasquez et al., 2011; Taylor et al., 2012).

Another limitation is that present analyses focused only on cortical mechanisms of facial affect recognition. Subcortical regions are clearly involved as well. For example, some studies have found functional amygdala abnormalities associated with poorer recognition performance in SZ (e.g., Li et al., 2010; Seyferth et al., 2009). MEG and EEG are capable of measuring deep structures such as hippocampus but only under certain circumstances (e.g., Hanlon et al., 2011; Williams, Nuechterlein, Subotnik, \& Yee, 2011).

The present findings suggest a set of neural mechanisms contributing to a deficit in facial affect processing in schizophrenia. Dynamic emotional faces revealed a sequence of alpha power modulation and alpha-modulated transregional connectivity that was lacking in schizophrenia. This impairment unfolded on a baseline of abnormal alpha and abnormal connectivity as well. Further research is needed to determine whether and how these neural oscillatory abnormalities contribute to impaired emotional face processing in schizophrenia. Fundamentally dysfunctional alpha regulation, evident under restingstate or prestimulus baseline conditions, may reflect dysfunctional information and input sampling (Jensen \& Mazaheri, 2010; Klimesch, 2012), which subsequently modifies inputspecific processing such as the recognition of unfolding affect in another person's face. The topography of dysfunctional alpha modulation may vary with the input and the task, whereas the functional significance of alpha oscillations is related to the modulation of input sampling. In compromising facial affect recognition, dysfunctional alpha modulation may be part of a cascade that eventually fosters dysfunctional socio-emotional processing (Taylor \& MacDonald, 2012) with consequences for higher socio-cognitive functions found to be deficient in schizophrenia.

Author Note This research was supported by the Deutsche Forschungsgemeinschaft (Grant No. Ro805/14 2). The authors report no conflict of interest. We thank Nathan Weisz for advice on data analyses, and Ursel Lommen and David Schubring for assistance with data collection.

\section{References}

Ackenheil, M., Stotz Ingenlath, G., Dietz Bauer, R., \& Vossen, A. (1999). M.I.N.I.: Mini International Neuropsychiatric Interview (German Version 5.0.0 DSM IV). Munich, Germany: Psychiatrische Universitätsklinik München.

Adolphs, R., Damasio, H., Tranel, D., Cooper, G., \& Damasio, A. R. (2000). A role for somatosensory cortices in the visual recognition of emotion as revealed by three dimensional lesion mapping. Journal of Neuroscience, 20, 26832690.

Alfimova, M. V., Abramova, L. I., Barhatova, A. I., Yumatova, P. E., Lyachenko, G. L., \& Golimbet, V. E. (2009). Facial affect recog nition deficit as a marker of genetic vulnerability to schizophrenia. Spanish Journal of Psychology, 12, 4655.

Bachmann, P., Kim, J., Yee, C. M., Therman, S., Manninen, M., Lönnquist, J., \& Cannon, T. D. (2008). Abnormally high EEG alpha synchrony during working memory maintenance in twins discordant for schizophrenia. Schizophrenia Research, 103, 29 297. doi:10.1016/j.schres.2008.04.006

Bastiaansen, M. C., \& Knosche, T. R. (2000). Tangential derivative mapping of axial MEG applied to event related desynchronization research. Clinical Neurophysiology, 111, 1300 1305. doi:10.1016/ S1388 2457(00)00272 8

Behrmann, M., \& Plaut, D. C. (2013). Distributed circuits, not circumscribed centers, mediate visual recognition. Trends in Cog nitive Neuroscience, 17, 210 219. doi:10.1016/j.tics.2013.03.007

Besche Richard, C., Bourrin Tisseron, A., Olivier, M., Cuervo Lombard, C. V., \& Limosin, F. (2012). Recognition of facial emotions and theory of mind in schizophrenia: Could the theory of mind deficit be due to the non recognition of facial emotions? Encephale, 38, 241 247. doi:10.1016/j.encep.2011.04.006

Bora, E., Yucel, M., \& Pantelis, C. (2009). Theory of mind impairment in schizophrenia: Meta analysis. Schizophrenia Research, 109, 1 9. doi:10.1016/j.schres.2008.12.020

Bullmore, E., \& Sporns, O. (2009). Complex brain networks: Graph theoretical analysis of structural and functional systems. Nature Reviews Neuroscience, 10, 186 198. doi:10.1038/nrn2575

Chan, R. C., Li, H., Cheung, E. F., \& Gong, Q. Y. (2010). Impaired facial emotion perception in schizophrenia: A meta analysis. Psychiatry Research, 178, 381 390. doi:10.1016/j.psychres.2009.03.035

Delvecchio, G., Sugranyes, G., \& Frangou, S. (2013). Evidence of diagnostic specificity in the neural correlates of facial affect pro cessing in bipolar disorder and schizophrenia: A meta analysis of functional imaging studies. Psychological Medicine, 43, 553569. doi: $10.1017 /$ S00333291712001432

Falkenberg, I., Bartels, M., \& Wild, B. (2008). Keep smiling! Facial reactions to emotional stimuli and their relationship to emotional contagion in patients with schizophrenia. European Archives of Psychiatry and Clinical Neuroscience, 258, 245253.

Fichtenholz, H. M., Hopfinger, J. B., Graham, R., Detwiler, J. M., \& LaBar, K. S. (2009). Event related potentials reveal temporal staging of dynamic facial expression and gaze shift effects on attentional orienting. Social Neuroscience, 4, 317 331. doi:10. 1080/1470910902809487

Fisher, N. I. (1993). Statistical analysis of circular data. Cambridge, UK: Cambridge University Press.

Fiszdon, J. M., \& Bell, M. D. (2009). Effects of presentation modality and valence on affect recognition performance in schizophrenia and healthy controls. Psychiatry Research, 170, 114 118. doi:10. 1016/j.psychres.2008.11.014

Frith, C. D., \& Corcoran, R. (1996). Exploring "theory of mind" in people with schizophrenia. Psychological Medicine, 26, 521530. doi:10.1017/S0033291700035601

Frith, C. D., \& Frith, U. (1999). Interacting minds A biological basis. Science, 286, 16921695. 
Garrido Vasquez, P., Jessen, S., \& Kotz, S. (2011). Perception of emotion in psychiatric disorders: On the possible role of task, dynamics, and multimodality. Social Neuroscience, 6, 515536. doi:10.1080/17470919.2011.620771

Gross, J., Kujala, J., Hamalainen, M., Timmermann, L., Schnitzler, A., \& Salmelin, R. (2001). Dynamic imaging of coherent sources: Studying neural interactions in the human brain. Proceedings of the National Academy of Sciences, 98, 694 699. doi:10.1073/pnas.98.2.694

Haegens, S., Nacher, V., Luna, R., Romo, R., \& Jensen, O. (2011). Alpha oscillations in the monkey sensorimotor network influence discrimination performance by rhythmical inhibition of neuronal spiking. Proceedings of the National Academy of Sciences, 108, 19377 19382. doi:10.1073/pnas.1117190108

Hall, J., Whalley, H. C., McKirdy, J. W., Romaniuk, L., McGonigle, D., McIntosh, A. M., \& Lawrie, S. M. (2008). Overactivation of fear systems to neutral faces in schizophrenia. Biological Psychiatry, 64, 70 73. doi:10.1016/j.biopsych.2007.12.014

Hämäläinen, M., Hari, R., Ilmoniemi, R. J., Knuutila, J., \& Lounasmaa, O. V. (1993). Magnetoencephalography: Theory, instrumentation, and applications to noninvasive studies of the working human brain. Reviews of Modern Physics, 65, 413497.

Hanlon, F. M., Houck, J. M., Pyeatt, C. J., Lundy, L. S., Euler, M. J., Weisend, M. P., \& Tesche, C. D. (2011). Bilateral hippocampal dysfunction in schizophrenia. NeuroImage, 58, 11581168. doi:10.1016/j.neuroimage.2011.06.091

Hanslmayr, S., Gross, J., Klimesch, W., \& Shapiro, K. L. (2011). The role of alpha oscillations in temporal attention. Brain Research Reviews, 67, 331 343. doi:10.1016/j.brainresrev.2011.04.002

Hars, M., Hars, M., Stam, C. J., \& Calmets, C. (2011). Effects of visual context upon functional connectivity during observation of bio logical motions. PLoS One, 6, 225903.

Harvey, P. O., Lee, J., Cohen, M. S, Engel, S. A., Glahn, D. C., Nuechterlein, K. H., \& Green, M. F. (2011). Altered dynamic cou pling of lateral occipital complex during visual perception in schizo phrenia. Neurolmage, 55, 1219 1226. doi:10.1016/j.neuroimage. 2010.12.045

Higashima, M., Tsujada, T., Nagasawa, T., Oka, T., Okamoto, T., Okamoto, Y., \& Koshino, Y. (2007). Reduction in event related alpha attenuation during performance of an auditory oddball task in schizophrenia. International Journal of Psychophysiology, 65, 95 102. doi:10.1016/j.ijpsycho.2007.03.008

Hinkley, L. B., Vinogradov, S., Guggisberg, A. G., Fisher, M., Findlay, A. M., \& Nagarajan, S. S. (2011). Clinical symptoms and alpha band resting state functional connectivity imaging in patients with schizophrenia: Implications for novel approaches for treatment. Biological Psychiatry. doi:10.1016/j.biopsych.2011.06.029

Hofer, A, Benecke, C., Edlinger, M., Huber, R., Kemmler, G., Rettenbacher, M. A., \& Fleischhacker, W. (2009). Facial emotion recognition and its relationship to symptomatic, subjective, and functional outcomes in outpatients with chronic schizophrenia. Eu ropean Psychiatry, 24, 27 32. doi:10.1016/j.eurpsy.2008.06.008

Iacoboni, M., \& Dapretto, M. (2006). The mirror neuron system and the consequences of its dysfunction. Nature Reviews Neuroscience, 7 , 942 951. doi:10.1038/nrn2024

Ikezawa, K., Ishii, R., Iwase, M., Kurimoto, R., Canuet, L., Takahashi, H., \& Takeda, M. (2011). Decrease alpha event related synchro nization in the left posterior temporal cortex in schizophrenia: A magnetoencephalographic beamformer study. Neuroscience Re search, 71, 235 243. doi:10.1016/j.neures.2011.07.1819

Irani, F., Seligman, S., Kamath, V., Kohler, C., \& Gur, R. C. (2012). A meta analysis of emotion perception and functional outcomes in schizophrenia. Schizophrenia Research, 137, 203 211. doi:10. 1016/j.schres.2012.01.023

Jensen, O., Bonneford, M., \& Van Rullen, R. (2012). An oscillatory mechanism for prioritizing salient unattended stimuli. Trends in Cognitive Sciences, 16, 200 206. doi:10.1016/j.tics.2012.03.002
Jensen, O., \& Mazaheri, A. (2010). Shaping functional architecture by oscillatory alpha activity: Gating by inhibition. Frontiers in $H u$ man Neuroscience, 4, 186. doi:10.3389/fnhum.2010.00177

Johnstone, P. J., Enticott, P. G., Mayes, A. K., Hoy, K. E., Herring, S. E., \& Fitzgerald, P. B. (2010). Symptom correlates of static and dynamic affect processing in schizophrenia: Evidence of a double dissociation? Schizophrenia Bulletin, 36, 680 687. doi:10.1093/ schbul/sbn136

Jung, H. T., Kim, D. W., Kim, S., Im, C. H., \& Lee, S. H. (2012). Reduced source activity of event related potentials for affective facial pictures in schizophrenia patients. Schizophrenia Research, 136, 150 159. doi:10.1016/j.schres.2011.10.023

Kay, S. R., Fiszbein, A., \& Opler, L. A. (1987). The positive and negative syndrome scale (PANSS) for schizophrenia. Schizophre nia Bulletin, 13, 261276.

Keil, J., Mueller, N., Ihssen, N., \& Weisz, N. (2012). On the variability of the McGurk effect: Audiovisual integration depends on prestimulus brain states. Cerebral Cortex, 22, 221 231. doi:10. 1093/cercor/bhr125

Keil, J., Weisz, N., Paul Jordanov, I., \& Wienbruch, C. (2010). Local ization of the magnetic equivalent of the ERN and induced oscil latory brain activity. NeuroImage, 51, 404 411. doi:10.1016/j. neuroimage.2010.02.003

Klimesch, W. (2012). Alpha band oscillations, attention, and controlled access to stored information. Trends in Cognitive Sciences, 16, 606 617. doi:10.1016/j.tics.2012.10.007

Klimesch, W., Sauseng, P., \& Hanslmayr, S. (2007). EEG alpha oscil lations: The inhibition timing hypothesis. Brain Research Re views, 53, 63 88. doi:10.1016/j.brainresrev.2006.06.003

Koh, Y., Shin, K. S., Choi, J. S., Kang, D. H., Jang, J. H., Cho, K. H., \& Kwon, J. S. (2011). An MEG study of alpha modulation in patients with schizophrenia and in subjects at high risk of developing psychosis. Schizophrenia Research, 126, 36 42. doi:10.1016/j. schres.2010.10.001

Kohler, C. G., Walker, J. B., Martin, E. A., Healey, K. M., \& Moberg, P. J. (2010). Facial emotion perception in schizophrenia: A meta analytic review. Schizophrenia Bulletin, 36, 1009 1919. doi:10. 1093/schbul/sbn192

Krakowski, A. I., Ross, L. A., Snyder, A. C., Sehatpour, P., Kelly, S. P., \& Foxe, J. J. (2011). The neurophysiology of human biological motion processing: A high density electrical mapping study. NeuroImage, 56, 373 383. doi:10.1016/j.neuroimage.2011.01.058

Kurtz, M. M., \& Richardson, C. L. (2011). Social cognitive training for schizophrenia: A meta analytic investigation of controlled re search. Schizophrenia Bulletin, 38, 1092 1104. doi:10.1093/ schbul/sbr036

Lachaux, J. P., Rodriguez, E., Martinerie, J., \& Varela, F. J. (1999). Measuring phase synchrony in brain signals. Human Brain Map ping, 8, 194208.

Lang, P. J. (1979). Presidential address, 1978. A bio informational theory of emotional imagery. Psychophysiology, 16, 495512.

Langner, O., Dotsch, R., Bijlstra, G., Wigboldus, D. H. J., Hawk, S. T., \& van Knippenberg, A. (2010). Presentation and validation of the Radboud Faces Database. Cognition \& Emotion, 24, 13771388. doi:10.1080/02699930903485076

Leitman, D. J., Loughead, J., Wolf, D. H., Ruparel, K., Kohler, C. G., Elliott, M. A., \& Gur, R. C. (2008). Abnormal superior temporal connectivity during fear perception in schizophrenia. Schizophre nia Bulletin, 34, 673 678. doi:10.1093/schbul/sbn052

Leppänen, J. M., Niehaus, D. J., Koen, L., DuToit, E., Schoenman, R , \& Emsey, R. (2006). Emotional face processing deficit in schizophre nia: A replication study in a South African Xhosa population. Schizophrenia Research, 84, 323 330. doi:10.1016/j.schres.2006. 02.007

Li, H. J., Chan, R. C., Gong, Q. Y., Liu, S. M., Shum, D., \& Ma, Z. L. (2012). Facial emotion processing in patients with schizophrenia 
and their non psychotic siblings: A functional magnetic resonance imaging study. Schizophrenia Research, 134, 143 150. doi:10. 1016/j.schres.2011.10.019

Li, H., Chan, R. C., McAlonan, G. M., \& Gong, Q. Y. (2010). Facial emotion processing in schizophrenia: A meta analysis of function al neuroimaging data. Schizophrenia Bulletin, 36, 10291039. doi:10.1093/schbul/sbn 190

Lynn, S. K., \& Salisbury, D. F. (2008). Attenuated modulation of the N170 ERP by facial expressions in schizophrenia. Clinical EEG and Neuroscience, 39, 108111.

Mandal, M. K., Pandey, R., \& Prasad, A. B. (1998). Facial expression of emotions and schizophrenia: A review. Schizophrenia Bulletin, 24, 399412.

Maris, E., \& Oostenveld, R. (2007). Nonparametric statistical testing of EEG and MEG data. Journal of Neuroscience Methods, 164, 177 190. doi:10.1016/jneumeth.2007.03.024

Mayes, A. K., Pipingas, A., Silberstein, R. B., \& Johnston, P. (2009). Steady state visually evoked potential correlates of static and dynamic emotional face processing. Brain Topography, 22, 145 157. doi:10.1007/s10548 00901065

Miller, G. A., Crocker, L. D., Spielberg, J. M., Infantolino, Z. P., \& Heller, W. (2013). Issues in localization of brain function: The case of lateralized frontal cortex in cognition, emotion, and psychopa thology. Frontiers in Integrative Neuroscience, 7, 2. doi:10.3389/ fnint.2013.00002

Mueser, K., Penn, D. L., Blanchard, J. J., \& Bellack, A. S. (1997). Affect recognition in schizophrenia: A synthesis of findings across three studies. Psychiatry, 1, 301308.

Mukherjee, P., Whalley, H. C., McKirdy, J. W., McIntosh, A. M., Johnstone, E. C., Lawrie, S. M., \& Hall, J. (2012). Lower effective connectivity between amygdala and parietal regions in response to fearful faces in schizophrenia. Schizophrenia Research, 134, 118 124. doi:10.1016/j.schres.2011.09.033

Niedenthal, P. M. (2007). Embodying emotion. Science, 316, 1002 1005. doi:10.1126/science. 1136930

Nikulin, V. V, Jönsson, E. G., \& Brismar, T. (2012). Attenuation of long range temporal correlations in the amplitude dynamics of alpha and beta neuronal oscillations in patients with schizophrenia. NeuroImage, 61, 162 169. doi:10.1016/j.neuroimage.2012.03.008

Norton, D., McBain, R., Holt, D. J., Ongur, D., \& Chen, Y. (2009). Association of impaired facial affect recognition with basic facial and visual processing deficits in schizophrenia. Biological Psychi atry, 65, 1084 1098. doi:10.1016/j.biopsych.2009.01.026

Oldfield, R. C. (1971). The assessment and analysis of handedness: The Edinburgh inventory. Neuropsychologia, 9, 97 113. doi:10.1016/ $00283932(71) 900674$

Oostenveld, R., Fries, P., Maris, E., \& Schoffelen, J. M. (2011). FieldTrip: Open source software for advanced analysis of MEG, EEG, and invasive electrophysiological data. Computational In telligence and Neuroscience, 2011, 156869. doi:10.1155/2011/ 156869

Pineda, J. A. (2005). The functional significance of mu rhythms: Trans lating "seeing" and "hearing" into "doing. Brain Research Re views, 50, 57 68. doi:10.1016/j.brainresrev.2005.04.005

Pineda, J. A., \& Hecht, E. (2009). Mirroring and mu rhythm involve ment in social cognition: Are there dissociable subcomponents of theory of mind? Biological Psychology, 80, 306 314. doi:10. 1016/j.biopsycho.2008.11.003

Pinkham, A. E., Gur, R. E., \& Gur, R. C. (2007). Affect recognition deficits in schizophrenia: Neural substrates and psychopharmaco logical implications. Expert Review of Neurotherapeutics, 7, 807 816. doi:10.1586/14737175.7.7.807

Pitcher, D., Garrido, L., Walsh, V., \& Duchaine, B. C. (2008). Trans cranial magnetic stimulation disrupts the perception and embodi ment of facial expressions. Journal of Neuroscience, 28, 8929 8933. doi:10.1523/jneurosci.1450 08.2008
Pomarol Clotet, E., Hynes, F., Ashwin, C., Bullmore, E. T., McKenna, P. J., \& Laws, K. R. (2010). Facial emotion processing in schizo phrenia: A non specific neuropsychological deficit? Psychologi cal Medicine, 40, 911 919. doi:10.1017/S0033291709991309

Popov, T., Iordanov, T., Weisz, N., Elbert, T., Rockstroh, B., \& Miller, G. A. (2011). Evoked and induced oscillatory activity contributes to abnormal sensory gating in schizophrenia. NeuroImage, 56, 307 314. doi:10.1016/j.neuroimage.2011.02.016

Popov, T., Miller, G. A., Rockstroh, B., \& Weisz, N. (2013). Modula tion of alpha power and functional connectivity during facial affect recognition. Journal of Neuroscience, 33, 6018 6026. doi:10. 1523/jneurosci.2763 12.2013

Ramos Loyo, J., Gonzales Garrido, A. A., Sanchez Loyo, L. M., Medina, V., \& Basar Eroglu, C. (2009). Event related potentials and event related oscillations during identity and facial emotional processing in schizophrenia. International Journal of Psychophysiology, 71, 84 90. doi:10.1016/j.ijpsycho.2008.07.008

Rosnow, R., \& Rosenthal, R. (2003). Effect sizes for experimenting psychologists. Canadian Journal of Experimental Psychology, 57, 221 237. doi:10.1037/h0087427

Rubinov, M., \& Sporns, O. (2010). Complex network measures of brain connectivity: Uses and interpretations. NeuroImage, 52, 1059 1069. doi:10.1016/j.neuroimage.2009.10.003

Sato, W., Kochiyama, T., Yoshikawa, S., Naito, E., \& Matsumura, M. (2004). Enhanced neural activity in response to dynamic facial expressions of emotion: An fMRI study. Brain Research, 20, 81 91.

Satterthwaite, T. D., Wolf, D. H., Loughead, J., Ruparel, K., Valdez, J. N., Siegel, S. J., \& Gur, R. C. (2010). Association of enhanced limbic response to threat with decreased cortical facial recognition memory response in schizophrenia. The American Journal of Psychiatry, 167, 418 426. doi:10.1176/appi.ajp.2009.09060808

Seyferth, N. Y., Pauly, K., Kellermann, T., Shah, N. J., Ott, G., Herpertz Dahlmann, B., \& Hable, U. (2009). Neuronal correlates of facial emotion discrimination in early onset schizophrenia. Neuropsychopharmacology, 34, 477 487. doi:10.1038/npp.2008. 93

Silver, H., Bilker, W., \& Goodman, C. (2009). Impaired recognition of happy, sad and neutral expressions in schizophrenia is emotion, but not valence, specific and context dependent. Psychiatry Re search, 169, 101106. doi:10.1016/j.psychres.2008.11.017

Simon, D., Craig, K. D., Miltner, W. H., \& Rainville, P. (2006). Brain responses to dynamic facial expressions of pain. Pain, 126, 309 318 .

Singh, F., Pineda, J., \& Cadenhead, K. (2011). Association of impaired EEG mu wave suppression, negative symptoms and social func tioning in biological motion processing in first episode of psycho sis. Schizophrenia Research, 130, 182 186. doi:10.1016/i.schres. 2011.04004

Streit, M., Ionanides, A., Sinnemann, T., Wölwer, W., Dammers, J., Zilles, K., \& Gaebel, W. (2001). Disturbed facial affect recognition in patients with schizophrenia associated with hypoactivity in distributed brain regions: A magnetoencephalographic study. The American Journal of Psychiatry, 158, 1429 1435. doi:10.1176/ appi.ajp.158.9.1429

Sugranyes, G., Kyriakopoulos, M., Corrigall, R., Taylor, E., \& Frangou, S. (2011). Autism spectrum disorders and schizophrenia: Meta analysis of the neural correlates of social cognition. PLoS One, 6, e25322.

Taylor, S. F., Kang, J., Brege, I. S., Tso, I. F., Hosanagar, A., \& Johnson, T. D. (2012). Meta analysis of functional neuroimaging studies of emotion perception and experience in schizophrenia. Biological Psychiatry, 71, 136 145. doi:10.1016/j.biopsych.2011.09.007

Taylor, S. F., \& MacDonald, A. W., III. (2012). Brain mapping bio markers of socio emotional processing in schizophrenia. Schizo phrenia Bulletin, 38, 73 80. doi:10.1093/schbul/sbr105 
Thompson, J. C., Hardee, J. E., Panayiotou, A., Crewther, D., \& Puce, A. (2007). Common and distinct brain activation to viewing dy namic sequences of face and hand movements. NeuroImage, 37, 966 973. doi:10.1016/j.neuroimage.2007.05.058

Turetsky, B. I., Kohler, C. G., Indersmitten, T., Bhati, M. T., Charbonnier, D., \& Gur, R. C. (2007). Facial emotion recognition in schizophrenia: When and why does it go awry? Schizophrenia Research, 94, 253 263. doi:10.1016/i.schres.2007.05001

Uhlhaas, P., Haenschel, C., Nikolic, D., \& Singer, W. (2008). The role of oscillations and synchrony in cortical networks and their puta tive relevance for the pathophysiology of schizophrenia. Schizo phrenia Bulletin, 34, 927 943. doi:10.1093/schbul/sbn062

Uhlhaas, P., \& Singer, W. (2010). Abnormal neural oscillations and synchrony in schizophrenia. Nature Reviews Neuroscience, 11, 100 113. doi:10.1038/nm2774

van der Gaag, C., Mindera, R. B., \& Keysers, C. (2007). Facial expres sions: What the mirror neuron system can and cannot tell us. Social Neuroscience, 2, 179 222. doi:10.1080/17470910701376878

Williams, J. J., Nuechterlein, K. H., Subotnik, K. L., \& Yee, C. M. (2011). Distinct neural generators of sensory gating in schizophrenia.
Psychophysiology, 48, 470 478. doi:10.1111/j.1469 8986.2010. 01119.x

Winograd Gurvich, C., Fitzgerald, P. B., Georgiou Karistianis, N., Millist, L., \& White, O. (2008). Inhibitory control and spatial working memory: A saccadic eye movement study of negative symptoms in schizophrenia. Psychiatry Research, 15, 919. doi:10.1016/j.psychres.2007.02.004

Wölwer, W., Brinkmeyer, J., Stroth, S., Streit, M., Bechdolf, A., Ruhrmann, S., \& Gaebel, W. (2012). Neurophysiological corre lates of impaired facial affect recognition in individuals at risk for schizophrenia. Schizophrenia Bulletin, 38, 1021 1029. doi:10. 1039/schbul/sbr013

Wynn, J. K., Jahshan, C., Altshuler, L. L., Glahn, D. C., \& Green, M. F. (2013). Event related potential examination of facial affect pro cessing in bipolar disorder and schizophrenia. Psychological Med icine, 43, 109 117. doi:10.1017/S0033291712001006

Wynn, J. K., Lee, J., Horan, W. P., \& Green, M. F. (2008). Using event related potential to explore stages of facial affect recognition deficits in schizophrenia. Schizophrenia Bulletin, 34, 679687. doi: $10.1093 / \mathrm{schbul} / \mathrm{sbn} 047$ 